\title{
Die thüringische Landtagswahl vom 30. August 2009: Desaster für Althaus-CDU mündet in schwarz-rotem Bündnis
}

\author{
Heiko Gothe
}

Der Ausgang der Landtagswahl in Thüringen am 30. August ist in mehrerlei Hinsicht bemerkenswert. Das Ergebnis bedeutete für die CDU ein Desaster, den Verlust ihrer dominanten Stellung im thüringischen Parteiensystem und den Gang in ein Bündnis mit der SPD unter Führung der ersten CDU-Ministerpräsidentin Deutschlands. Der SPD fiel trotz mäßigen Ergebnisses die Rolle des „Königsmachers“ zu. Die Hoffnung einer erneut erstarkten Linken, erstmals einen Ministerpräsidenten stellen zu können, erfüllte sich nicht, weil die SPD nach turbulenter Sondierungsphase die CDU als Koalitionspartner vorzog. Das Parteiensystem erweiterte sich auf fünf Parteien, weil FDP und Grüne nach 15 Jahren wieder den Einzug in den Landtag schafften.

\section{Die Ausgangslage}

Bei der Landtagswahl 2004 konnte die CDU trotz Verlusten mit einem Wähleranteil von 43,0 Prozent (-8,0 Punkte) ihre absolute Mehrheit im Landesparlament knapp verteidigen. Die SPD musste mit 14,5 Prozent das bis dahin zweitschlechteste Ergebnis bei Landtagswahlen in der Bundesrepublik verkraften. Die damalige PDS baute mit 26,1 Prozent $(+4,8)$ ihre Stellung als zweitstärkste Partei aus. Sowohl die Grünen (4,5 Prozent) als auch die FDP (3,6 Prozent) vermochten trotz Zuwächsen nicht, die Fünfprozenthürde zu überspringen. Die Wahlbeteiligung war auf ein Rekordtief gefallen (53,8 Prozent). ${ }^{1}$

Konnte Ministerpräsident Dieter Althaus 2004 die Popularitätsschwäche der CDU noch kompensieren, gelang ihm dies in der folgenden Wahlperiode immer weniger. Spätestens zur Regierungsumbildung im Mai 2008 galt der „Ziehsohn“ Bernhard Vogels als angeschlagen. Die Kabinettsumbildung 2 , mit der Althaus für „neue Impulse sorgen“ und „verlorenes

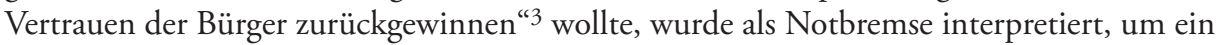
gutes Jahr vor der Landtagswahl den sinkenden Umfragewerten entgegenzuwirken. ${ }^{4}$ War

1 Vgl. Karl Schmitt, Die thüringische Landtagswahl vom 13. Juni 2004: Glückliche Bestätigung eines gelungenen Stabwechsels, in: ZParl, 37. Jg. (2006), H. 1, S. 126 - 144.

2 Anlass war der Rücktritt des damaligen Innenministers Karl Heinz Gasser, der die Konsequenzen aus der gescheiterten Polizeireform zog. Bereits die Diskussion um die Neubesetzungen zwang den Regierungschef in die Defensive: Als sich herausstellte, dass sein Favorit für das Kultusministerium, Peter Krause, 1998 einige Monate als Redakteur für die rechtsgerichtete Wochenzeitung "Junge Freiheit" arbeitete und die designierte Justizministerin Marion Walsmann als DDR-Volkskammerabgeordnete eine Solidaritätsbekundung an die chinesische Führung nach der Niederschlagung der Studentenunruhen 1989 mitgetragen hatte, verstärkte sich der Gegenwind. Walsmann distanzierte sich von ihrer Entscheidung und wurde trotzdem Justizministerin, die Nominierung Krauses hingegen wurde nach massiver öffentlicher Kritik zurückgezogen.

3 Sven Heitkamp, Aus dem Tritt geraten, in: Die Welt vom 3. Mai 2008, S. 4.

4 Die CDU war im Mai 2008 in den Umfragen auf 33 Prozent abgesackt. Vgl. Infratest dimap, ThüringenTREND Mai 2008, Berlin 2008. 
Althaus damit gut ein Jahr vor der Landtagswahl politisch angeschlagen, sorgte der von ihm durch einen Fahrfehler verursachte Skiunfall am Neujahrstag 2009, bei dem eine 41-jährige Mutter starb und er selbst ein Schädel-Hirn-Trauma erlitt, für ein weiteres, in der politischen Wirkung schwer kalkulierbares Handicap. In den Monaten nach dem Unfall war zunächst nicht klar, ob und wann er seine Amtsgeschäfte wieder aufnehmen und die Thüringer CDU in den Wahlkampf führen würde. Im April kehrte Althaus in die Kurmainzische Statthalterei zurück, mit einer Anerkennung seiner Schuld tat er sich jedoch schwer. ${ }^{5}$

Auch die Sozialdemokraten hatten auf personeller Ebene Hürden zu überwinden. Richard Dewes ${ }^{6}$ versuchte, dem Landesvorsitzenden Christoph Matschie die Spitzenkandidatur streitig zu machen. Dewes trat für ein Regierungsbündnis mit der Linken ein, auch wenn die SPD in dieser Konstellation der Juniorpartner sein sollte. Matschie wollte lediglich mit den Linken koalieren, wenn die SPD bei der Wahl mehr Stimmen erhalten und somit den Ministerpräsidenten stellen würde. Die Entscheidung zugunsten Matschies wurde im Februar 2008 von der Parteibasis getroffen: In einer Urwahl votierten 71,6 Prozent der SPD-Mitglieder für ihn als Spitzenkandidaten, Dewes erhielt lediglich 27,0 Prozent. ${ }^{7}$ Beim vorgezogenen Parteitag im Juni 2008 wurde Matschie mit 75 Prozent der Stimmen als Landesvorsitzender bestätigt.

Von der Bundespolitik waren wegen des konfrontationsarmen Vorwahlkampfes im Bund nur wenige Impulse für die Landesentscheidung zu erwarten. Allenfalls FDP und Grüne konnten sich vor dem Hintergrund des Europawahlergebnisses Hoffnung auf ein gutes Abschneiden am 30. August machen. ${ }^{8}$ CDU und Linke hatten am 7. Juni hingegen Einbußen, die SPD nur leichte Zugewinne zu verzeichnen. Allerdings stand für eine Mehrheit der Thüringer ohnehin die Landespolitik im Vordergrund ihrer Wahlentscheidung. ${ }^{9}$

\section{Der Wahlkampf}

Der Wahlkampf verlief sehr ruhig. Es fehlten polarisierende und emotional besetzte Sachthemen, die die Auseinandersetzung hätten spannend werden lassen können. Umso mehr wurden die Wochen vor dem Urnengang von der Diskussion um Althaus' tragischen Skiunfall und seinen Umgang damit bestimmt. Die politischen Akteure waren sich zwar einig, dass das Unglück kein Wahlkampfthema werden sollte, Althaus zog aber dennoch Kritik

5 Ein österreichisches Gericht verurteilte Althaus am 3. März wegen fahrlässiger Tötung zu einer Geldstrafe von 33.300 Euro und einer Schmerzensgeldzahlung von 5.000 Euro an den Witwer.

6 Dewes war während der ersten thüringischen CDU/SPD-Koalition von 1994 bis 1999 Innenminister und von 1996 bis 1999 SPD-Landesvorsitzender. Zu seinem Rücktritt nach der Landtagswahl 1999 siehe Karl Schmitt, Die Landtagswahlen in Brandenburg und Thüringen vom 5. und 12. September 1999: Landespolitische Entscheidungen im Schlagschatten der Bundespolitik, in: ZParl, 31. Jg. (2000), H. 1, S. 43 - 68, S. 66 f.

7 SPD Thüringen: Urwahl in Thüringen - Matschie gewinnt mit überwältigender Mehrheit, http://www.spd-thueringen.de/index.php?nr=3992\&menu=1 (Abruf am 19. November 2009). Von den rund 4.400 Mitgliedern beteiligten sich 63,2 Prozent an der Abstimmung.

8 FDP (8,2 Prozent) und Grüne (5,8 Prozent) hatten am 7. Juni wenig Mühe, die Fünfprozentmarke zu überspringen.

9 Vgl. Forschungsgruppe Wahlen, Wahl in Thüringen, Eine Analyse der Landtagswahl vom 30. August 2009, Mannheim 2009, S. 36. 
auf sich, weil er nicht persönlich bei seiner Partei und in der Öffentlichkeit auftrat, sondern vornehmlich über Boulevardmedien kommunizierte. Tatsächlich war der CDU-Politiker am 14. März in Abwesenheit zum Spitzenkandidaten für die Landtagswahl gewählt worden: „Die Partei beklatscht ein Phantom“"10, das 94,6 Prozent der Delegiertenstimmen bekam. Nach Auffassung politischer Beobachter setzte Althaus nun seinerseits den Skiunfall als Wahlkampfinstrument ein, indem er in Interviews über Besuche am Grab der Toten und das Entstehen eines freundschaftlichen Verhältnisses zu dem Witwer berichtete. Vier von zehn Bürgern (39 Prozent) schlossen sich der Auffassung an, er instrumentalisiere den Skiunfall für seine Wahlkampfziele. ${ }^{11}$ Althaus' Äußerungen sorgten auch auf Seiten des Witwers für Verärgerung, dessen Anwalt sie „...nicht nur als Vertrauensbruch, sondern als unangebracht und pietätlos bewertete..." ${ }^{2}$. Nach einer rund zehn Tage vor dem Wahltermin erwirkten „Stillschweigevereinbarung“ durfte sich Althaus nicht mehr zu Einzelheiten des Unfalls beziehungsweise zu möglicherweise die Familie des Opfers tangierenden Details äußern. ${ }^{13}$

Die CDU betonte in der ganz auf die Person Althaus zugeschnittenen Kampagne die aus ihrer Sicht herausragende Position des Freistaats unter den neuen Bundesländern in wirtschaftlicher, familien- und bildungspolitischer Hinsicht. Diese Erfolge wurden unmittelbar auf die CDU-geführte Landesregierung zurückgeführt. Tatsächlich wies Thüringen im Vergleich der neuen Bundesländer mit 11,5 Prozent im Juli 2009 die niedrigste Arbeitslosenquote und nach Sachsen die meisten Beschäftigten im verarbeitenden Gewerbe auf. Allerdings schwand der ehemals deutliche Vorsprung Thüringens gegenüber anderen Bundesländern: Bei der Entwicklung des Bruttoinlandsproduktes lag es 2008 nur noch auf Platz 12 aller Bundesländer - 2003 war es noch Platz 2. Auch das Problem der Abwanderung vor allem junger Menschen aufgrund fehlender Arbeitsplätze bestand nach wie vor.

Althaus hatte sich mehr oder weniger unbemerkt von dem Anspruch, die absolute Mehrheit zu verteidigen, verabschiedet und sprach zuletzt davon, „die Gestaltungsmehrheit“ für Thüringen unter seiner Führung zu erreichen. Er traf keine Koalitionsaussage, sondern wollte Verhandlungen vom Wahlergebnis abhängig machen.

Die Linke hingegen wollte Althaus ablösen und den ersten linken Ministerpräsidenten Deutschlands stellen. In ihrer Wahlkampagne stellte sie deshalb wie 2004 ihren Spitzenkandidaten Bodo Ramelow in den Mittelpunkt. Das Wahlprogramm enthielt die fünf „Leitprojekte" soziale Gerechtigkeit, Bildungschancen und kulturelle Teilhabe für alle, existenzsichernde Arbeitsplätze, eine Energieoffensive sowie mehr direkte Demokratie. Als Koalitionspartner sprach sie sich unter Berufung auf die größten politischen Gemeinsamkeiten für die SPD als Juniorpartnerin aus.

10 Philipp Neumann, Thüringens CDU beklatscht ein Phantom, in: Welt am Sonntag vom 15. März 2009, S. 6.

11 Falls nicht anders vermerkt, basieren alle vorgestellten Umfragergebnisse auf den Vorwahlerhebungen von Infratest dimap, siehe dazu Infratest dimap, Wahlreport Landtagswahl in Thüringen 30. August 2009, Berlin 2009, S. 22 ff.

12 Dirk Banse / Philipp Neumann, Althaus' Umgang mit Skiunfall wird Wahlkampfthema, in: Die Welt vom 22. August 2009, S. 2.

13 Vgl. Christiane Kohl, Althaus muss künftig zu Skiunfall schweigen, in: SZ vom 22./23. August 2009, S. 1. 
Die Sozialdemokraten wiederum, die erneut mit Christoph Matschie als Spitzenkandidat um Wählerstimmen warben, kündigten an, dieses Bündnis nur dann einzugehen, wenn sie den Ministerpräsidenten stellen können. Die SPD schloss aber auch eine Koalition mit der CDU nicht aus. Die Auseinandersetzung mit der Union konzentrierte sich auf den Vorwurf an die Regierungspartei, sich nach zehn Jahren Alleinregierung auf ihren Erfolgen auszuruhen. Daneben setzte die SPD inhaltliche Schwerpunkte mit ihrer Forderung nach Einführung eines gesetzlichen Mindestlohns, der Bildungs- und Energiepolitik mit dem Ziel des Ausbaus von Ganztagsschulen sowie der Förderung erneuerbarer Energien.

Zentrale Themen des FDP-Wahlkampfes waren die Arbeitsmarkt- und Mittelstandspolitik sowie die Forderung nach einer stabilen Finanzpolitik und einem einfacheren Steuersystem mit Stufentarif. Bildungspolitisch traten sie für ein einheitliches Abitur sowie für längeres gemeinsames Lernen bis zur 6. Klasse ein, wobei ein gegliedertes Schulsystem beibehalten werden sollte. Im Bereich der Sozialpolitik forderte die FDP die Einführung eines leistungsgerechten Bürgergeldes. Sie ging von einem Einzug ins Landesparlament aus und hielt eine Regierungsbeteiligung unter CDU-Führung für am realistischsten. Ihr Spitzenkandidat war wie 2004 der Landesvorsitzende Uwe Barth.

Auch die Grünen wollten mit ihrer alten und neuen Spitzenkandidatin Astrid RotheBeinlich die Fünfprozenthürde überspringen und damit die CDU-Regierung beenden. Inhaltlich stellten die Grünen - anders als 2004 - wieder verstärkt typisch grüne, ökologische Themen in den Mittelpunkt: Als Ziele formulierten sie, Thüringen bis 2050 komplett auf erneuerbare Energien umzustellen, die Verkehrspolitik auf den Ausbau des öffentlichen Verkehrsnetzes zu fokussieren, aber auch die Einführung eines Mindestlohns sowie eine bedingungslose Kindergrundsicherung. Ausgeschlossen wurde eine Koalition mit der regierenden CDU.

Die Grundstimmung im „grünen Herz Deutschlands“ fiel - trotz der aktuellen Finanzund Wirtschaftskrise - positiver aus als im Vorfeld der letzten Landtagswahl. Mehr als zwei Drittel der Thüringer waren nach eigenem Bekunden von der Krise bisher nicht persönlich betroffen. Vor diesem Hintergrund und als Folge der seit 2004 deutlich gesunkenen Arbeitslosenquote schätzten 31 Prozent der Bürger die wirtschaftliche Lage des Landes als sehr gut beziehungsweise gut ein, fünf Jahre zuvor waren es nur 16 Prozent. Gleichwohl waren zwei Drittel davon überzeugt, dass die ökonomische Situation in Thüringen weniger gut beziehungsweise schlecht sei. Ökonomische Themen wie die Arbeitslosigkeit (64 Prozent) und die Wirtschaftslage (19 Prozent), aber auch die Bildungspolitik (31 Prozent) standen auf der Problemagenda der Thüringer ganz oben. Unmittelbar vor der Wahl herrschte eine erkennbare Wechselstimmung: Jeder Zweite plädierte für eine Landesregierung unter neuer Führung (SPD 30 Prozent, Linke 19 Prozent). Lediglich 39 Prozent bevorzugten auch nach dem Wahltag eine unionsgeführte Regierung. Eine CDU-Alleinregierung schätzten nur noch 30 Prozent als gut für das Land ein, 14 Punkte weniger als fünf Jahre zuvor.

Zur Wahl des 5. Thüringer Landtages traten insgesamt neun Parteien an, so wenige wie noch nie seit der Bildung des Bundeslandes. Neben den drei Parlamentsparteien waren dies Bündnis90/Die Grünen, FDP, die Freien Wähler Thüringen, Republikaner, NPD und die ödp. CDU, Linke und SPD hatten in allen 44 Wahlkreisen Bewerber für die Direktmandate aufgestellt. Gleiches galt für die NPD, während FDP (41) und Grüne (35) nicht flächendeckend mit eigenen Kandidaten aufwarten konnten. 


\section{Das Wahlergebnis ${ }^{14}$}

Das Ergebnis der Landtagswahl in Thüringen am 30. August 2009 war ein Desaster für die regierende CDU und den Ministerpräsidenten Dieter Althaus. Die Regierungspartei blieb mit 31,2 Prozent der Zweitstimmen zwar stärkste Partei, büßte aber 11,7 Prozentpunkte ein und verspielte damit ihre Alleinregierung. Mit einem Minus von 104.789 Stimmen verlor sie rund ein Viertel ihrer Wähler. Im Vergleich zu 1990, als die CDU ihre Dominanz in Thüringen begründete, kam dies fast einer Halbierung gleich. Die christdemokratische Fraktion ist dramatisch geschrumpft: Von den 88 Abgeordneten im fünften Erfurter Landtag werden nur noch 30 (-15) von der Union gestellt. Während die CDU ihr schlechtestes Landtagswahlergebnis verkraften muss, legten nahezu alle anderen Parteien zu. Die Linke erzielte mit 27,4 Prozent $(+1,3)$ ihr bestes thüringisches Landesergebnis - und ihr zweitbestes Landtagswahlergebnis überhaupt. Allerdings kann sie nur noch 27 Abgeordnete stellen $(-1)$, weil die Zahl der Fraktionen von drei auf fünf angewachsen ist. Die SPD freute sich ebenfalls über Zugewinne (+4,1), blieb mit ihrem Ergebnis von 18,5 Prozent allerdings unter der 20-Prozentmarke. Die sozialdemokratische Fraktion vergrößert sich um drei auf 18 Mandatsträger. Nach fünfzehn Jahren parlamentarischer Abstinenz gelang der FDP und den Grünen wieder der Einzug in den Landtag. Die Liberalen vermochten ihre Stimmenzahl mehr als zu verdoppeln und sind mit 7,6 Prozent $(+4,0)$ viertstärkste Kraft im Land. Die Grünen erreichten 6,2 Prozent $(+1,6)$. Die Stimmenanteile der sonstigen Parteien (9,0 Prozent) markierten den höchsten Stand in der Landtagswahlgeschichte. Am stärksten war die rechtsradikale NPD, die trotz der Verdreifachung ihrer Stimmenzahl auf 4,3 Prozent an der Sperrklausel scheiterte. Den Freien Wählern gelang mit 3,9 Prozent ein Achtungserfolg. Als Folge dieses Wahlergebnisses hat sich das seit 1994 bestehende Dreiparteiensystem Thüringens zu einem „fluiden“ Fünfparteiensystem entwickelt, das durch eine größere Fragmentierung und erweiterte Koalitionsmöglichkeiten gekennzeichnet ist. ${ }^{15}$

\subsection{Die Wahlbeteiligung}

Anders als in Sachsen, wo der Wahlausgang im Vorfeld klar schien, stieg die Wahlbeteiligung in Thüringen an: 56,2 Prozent der Wahlberechtigten gaben ihre Stimme ab, 2,4 Punkte mehr als bei der Vorgängerwahl, was einem Plus von 20.095 Wählern entspricht.

Aufgrund der Koppelung mit der Bundestagswahl verzeichnete Thüringen 1994 den bisherigen Teilnahmerekord bei einer Landtagswahl. Die seitdem zu beobachtende Abnahme wurde 2009 gestoppt. Grund dafür dürfte der mehrheitlich verbreitete Wunsch nach einem politischen Wechsel gewesen sein, gepaart mit der Aussicht, eine Neuordnung der

14 Mit der Erststimme („Wahlkreisstimme“) wird der Wahlkreiskandidat in 44 Wahlkreisen gewählt, mit der Zweitstimme („Landesstimme“) die Landesliste einer Partei. Als Direktkandidat gewählt und im Landtag vertreten ist, wer die Mehrheit der Erststimmen im Wahlkreis erlangt (einfache Mehrheitswahl). An der Verteilung der weiteren Sitze nehmen nur die Parteien teil, die landesweit mindestens fünf Prozent der gültigen Zweitstimmen erhalten haben. In der Regel besteht das Parlament aus 88 Sitzen zuzüglich eventueller Überhang- und Ausgleichsmandate.

15 Vgl. Oskar Niedermayer, Die Entwicklung des bundesdeutschen Parteiensystems, in: Frank Decker I Viola Neu (Hrsg.), Handbuch der Deutschen Parteien, Wiesbaden 2007, S. 114 - 135, S. 126 ff. 
Tabelle 1: Ergebnis der Landtagswabl in Thüringen am 30. August 2009

\begin{tabular}{|c|c|c|c|c|c|c|c|c|c|}
\hline & \multicolumn{3}{|c|}{ Absolut } & \multicolumn{3}{|c|}{ Prozent } & \multicolumn{3}{|c|}{ Mandate } \\
\hline & 2009 & 2004 & Diff. & 2009 & 2004 & Diff. & 2009 & 2004 & Diff. \\
\hline Wahlberechtigte & 1.910 .074 & 1.958 .041 & -47.967 & & & & & & \\
\hline Wähler & 1.073 .651 & 1.053 .556 & +20.095 & 56,2 & 53,8 & $+2,4$ & & & \\
\hline Zweitstimme & & & & & & & & & \\
\hline Ungültige Stimmen & 19.354 & 42.978 & -23.624 & 1,8 & 4,1 & $-2,3$ & & & \\
\hline Gültige Stimmen & 1.054 .297 & 1.010 .578 & +43.719 & 98,2 & 95,9 & $+2,3$ & & & \\
\hline $\mathrm{CDU}$ & 329.302 & 434.088 & -104.786 & 31,2 & 43,0 & $-11,7$ & 30 & 45 & -15 \\
\hline Die Linke* & 288.915 & 263.717 & +25.198 & 27,4 & 26,1 & $+1,3$ & 27 & 28 & -1 \\
\hline SPD & 195.363 & 146.297 & +49.066 & 18,5 & 14,5 & $+4,1$ & 18 & 15 & +3 \\
\hline Grüne & 64.912 & 45.649 & +19.263 & 6,2 & 4,5 & $+1,6$ & 6 & - & +6 \\
\hline REP & 4.488 & 19.797 & -15.309 & 0,4 & 2,0 & $-1,5$ & - & - & - \\
\hline FDP & 80.600 & 36.483 & +44.117 & 7,6 & 3,6 & $+4,0$ & 7 & - & +7 \\
\hline Fr. Wähler Thüringen & 40.811 & 26.302 & +14.509 & 3,9 & 2,6 & $+1,3$ & - & - & - \\
\hline NPD & 45.451 & 15.695 & +29.756 & 4,3 & 1,6 & $+2,8$ & - & - & - \\
\hline ödp & 4.455 & 2.317 & +2.138 & 0,4 & 0,2 & $+0,2$ & - & - & - \\
\hline Sonstige & - & 20.233 & -20.233 & - & 2,0 & $-2,0$ & - & - & - \\
\hline
\end{tabular}

* Bis Landtagswahl 2004: PDS.

Anmerkung: Die Prozentpunktdifferenzen wurden mit ungerundeten Stimmenanteilen berechnet und können daher von den Veröffentlichungen des Landeswahlleiters abweichen.

Quelle: Thüringer Landesamt für Statistik.

politischen Verhältnisse im Land auch tatsächlich bewirken zu können. Allerdings liegt das Beteiligungsniveau aktuell trotzdem 18 Punkte unter dem von 1994.

Bemerkenswert ist, dass die Kluft zwischen Jung und Alt bei der demokratischen Teilhabe weiter auseinander gegangen ist. Während im Vergleich zu 2004 die Beteiligungsrate bei Jungwählern rückläufig war, ist sie bei über 60jährigen überdurchschnittlich angestiegen. Dies führt dazu, dass die Kluft zwischen den beiden Altersgruppen von rund 22 auf 28 Punkte angewachsen ist. ${ }^{16}$

\subsection{Wählerwanderung ${ }^{17}$}

Die Christdemokraten verloren als einzige Partei Stimmen an alle politischen Wettbewerber, am deutlichsten an die FDP (per Saldo minus 28.000) und an die SPD (-27.000), in geringerem Umfang aber auch an die Linke (-16.000). Die CDU war auch die einzige, bei

16 Eigene Berechnungen auf Basis der repräsentativen Wahlstatistik des Thüringer Landesamtes für Statistik.

17 Die Analysen zur Wählerwanderung und zum Wahlverhalten stützen sich auf eine Wählerbefragung in 200 repräsentativ ausgewählten Stimmbezirken. Dabei wurden das Stimmverhalten, das Alter und das Geschlecht von insgesamt 25.216 Wählern erhoben. 3.965 Wähler wurden ferner zu den Motiven ihrer Wahlentscheidung sowie zu weitergehenden sozialstrukturellen Merkmalen befragt. Da in der Wahltagsbefragung Briefwähler nicht berücksichtigt sind, wurden die Ergebnisse nach dem Endergebnis gewichtet, so dass Aussagen über die Gesamtheit der Wähler getroffen werden können. 
der mehr frühere Wähler den Urnen fernblieben als neue mobilisiert werden konnten (-6.000). Zudem litt die Union am stärksten durch verstorbene Wähler, was nur zur Hälfte durch die erfolgreiche Ansprache von Erstwählern wettgemacht werden konnte (-16.000). Der Linken gelang es, neben ehemaligen CDU-Wählern auch 5.000 Stimmen von der SPD abzuziehen. Durch die Mobilisierung ehemaliger Nichtwähler vermochte sie, die Verluste aufgrund der Überalterung ihrer Wählerschaft auszugleichen. Die Stimmenzuwächse der Sozialdemokraten beruhten neben dem Zustrom ehemaliger CDU-Wähler auf einer erfolgreichen Mobilisierung von Nichtwählern (+20.000) sowie in kleinerem Umfang von Zuzüglern (+7.000). Die Basis des FDP-Erfolgs war in erster Linie der Zustrom enttäuschter CDU-Wähler, aber auch die Mobilisierung ehemaliger Nichtwähler (+9.000) sowie die erfolgreiche Ansprache von Erstwählern (+4.000). Ähnliches - mit anderer Akzentuierung und auf niedrigerem Niveau - galt für die Grünen, die ebenfalls ehemalige Nichtwähler $(+8.000)$, frühere CDU-Wähler (+6.000) sowie Erstwähler $(+4.000)$ von sich überzeugen konnten. ${ }^{18}$

\subsection{Wahlverhalten nach Alter und Geschlecht}

Die Analyse des Wahlverhaltens in den verschiedenen Altersgruppen (vgl. Tabelle 2) zeigt den dramatischen Unterstützungsverlust für die CDU in den letzten Jahren. Bei den vorangegangenen beiden Landtagswahlen war sie noch mit komfortablem Abstand stärkste Kraft in allen Altersgruppen. 2009 hat sie mit Ausnahme der über 60-Jährigen überall zweistellige Verluste zu verzeichnen. Bei den 45- bis 59-Jährigen ist sie nicht mehr stärkste Partei. In dieser Altersgruppe haben die Christdemokraten binnen zehn Jahren ein Minus von 24 Punkten zu verzeichnen, in den anderen Altersgruppen sind es immer noch um die 20 Punkte. Einzig bei den über 60-Jährigen erreichte die CDU bei dieser Wahl noch ein Ergebnis über 30 Prozent. In den zahlenmäßig starken Altersgruppen ab 45 Jahren vermochte die Linke ihr Ergebnis auszubauen, so dass sie bei den 45- bis 59-Jährigen nun die meisten Stimmen auf sich vereinen kann und auch bei den über 60-Jährigen nur knapp hinter der Union platziert ist. Damit kann sie ihre leichten Verluste in den jüngeren Jahrgängen mehr als wettmachen. Neben der SPD, die über ein relativ ausgeglichenes Altersprofil verfügt, gelang es auch FDP und Grünen, sich in allen Altersgruppen zu verbessern. Beide kleineren Parteien weisen dabei ein ähnliches Altersprofil auf, das durch zweistellige Ergebnisse bei Wählern bis 45 Jahre und deutlich schwächere Resultate in den älteren Jahrgängen gekennzeichnet ist. Den Liberalen gelang es, ihre Stimmenanteile in den berufsaktiven Alterskohorten von 25 bis 59 Jahren zu verdoppeln. Auch die NPD legte durchweg zu. Sie überzeugte fast jeden zehnten Erst- und Jungwähler und erreichte auch noch in den Altersgruppen bis 44 Jahren Stimmenanteile über fünf Prozent. Der geringere Zuspruch bei älteren Wählern verhinderte den Landtagseinzug der Rechtsradikalen. Nach dem auch bei anderen Wahlen üblichen Muster ist der Rückhalt der NPD bei Männern größer als bei Frauen - in Thüringen dreimal so groß. Bei Männern von 18 bis 34 Jahren ist sie mit einem Anteil von elf Prozent sogar viertstärkste Kraft knapp vor den Grünen und nur wenig hinter der FDP. Auch die Freien Wähler legten in allen Altersgruppen zu.

18 Für eine detaillierte Darstellung der Wanderungsbewegungen siehe Infratest dimap, a.a.O. (Fn. 11), S. $10-16$. 
Tabelle 2: Wablverhalten nach Alter und Geschlecht 2009 und Veränderungen gegenüber 2004

\begin{tabular}{|c|c|c|c|c|c|c|c|c|c|c|c|c|c|c|}
\hline & \multicolumn{2}{|c|}{$\mathrm{CDU}$} & \multicolumn{2}{|c|}{ Die Linke* } & \multicolumn{2}{|c|}{ SPD } & \multicolumn{2}{|c|}{ FDP } & \multicolumn{2}{|c|}{ Grüne } & \multicolumn{2}{|c|}{ NPD } & \multicolumn{2}{|c|}{ FW } \\
\hline & $\%$ & Diff & $\%$ & Diff & $\%$ & Diff & $\%$ & Diff & $\%$ & Diff & $\%$ & Diff & $\%$ & Diff \\
\hline 18-24 Jahre & 25 & -11 & 21 & \pm 0 & 18 & +4 & 11 & +3 & 11 & +1 & 9 & +3 & 4 & +3 \\
\hline $25-34$ & 29 & -14 & 18 & -1 & 17 & +4 & 12 & +7 & 10 & +3 & 8 & +4 & 4 & +2 \\
\hline $35-44$ & 28 & -15 & 23 & -2 & 18 & +6 & 11 & +6 & 9 & +3 & 5 & +4 & 5 & +2 \\
\hline $45-59$ & 27 & -15 & 31 & +2 & 17 & +5 & 8 & +4 & 7 & +2 & 4 & +3 & 5 & +3 \\
\hline 60 und älter & 34 & -9 & 31 & +3 & 21 & +2 & 5 & +2 & 4 & +1 & 2 & +1 & 3 & +2 \\
\hline Frauen & 32 & -11 & 26 & \pm 0 & 21 & +5 & 7 & +4 & 7 & +2 & 2 & +1 & 4 & +3 \\
\hline 18-24 Jahre & 26 & -10 & 21 & -1 & 19 & +4 & 9 & +2 & 13 & +2 & 6 & +3 & 5 & +3 \\
\hline $25-34$ & 30 & -15 & 19 & -3 & 20 & +6 & 10 & +6 & 11 & +4 & 5 & +3 & 5 & +3 \\
\hline $35-44$ & 28 & -14 & 22 & -4 & 20 & +7 & 10 & +5 & 10 & +4 & 3 & +2 & 5 & +3 \\
\hline $45-59$ & 28 & -14 & 29 & \pm 0 & 19 & +6 & 8 & +4 & 7 & +2 & 2 & +2 & 5 & +3 \\
\hline 60 und älter & 38 & -8 & 27 & +2 & 22 & +3 & 5 & +2 & 4 & +1 & 1 & +1 & 3 & +2 \\
\hline Männer & 28 & -13 & 30 & +3 & 17 & +2 & 8 & +4 & 6 & +2 & 6 & +4 & 4 & +2 \\
\hline 18-24 Jahre & 25 & -11 & 21 & +2 & 17 & +3 & 12 & +4 & 9 & +1 & 11 & +3 & 4 & +2 \\
\hline $25-34$ & 28 & -14 & 18 & \pm 0 & 15 & +2 & 14 & +7 & 10 & +3 & 11 & +6 & 3 & +1 \\
\hline $35-44$ & 28 & -16 & 23 & \pm 0 & 15 & +4 & 11 & +7 & 9 & +3 & 8 & +5 & 4 & +2 \\
\hline $45-59$ & 26 & -15 & 33 & +3 & 15 & +3 & 8 & +4 & 6 & +2 & 6 & +5 & 5 & +3 \\
\hline 60 und älter & 30 & -10 & 36 & +5 & 20 & +1 & 5 & +2 & 3 & +1 & 3 & +2 & 3 & +1 \\
\hline
\end{tabular}

\subsection{Wahlverhalten nach Bildung, Beruf und Konfession}

Die Thüringen-CDU ist die Partei der einfachen Leute: Weil sie bei Wählern mit niedriger formaler Bildung ihre Verluste begrenzen konnte, erreichten die Christdemokraten hier mit 40 Prozent ihre besten Werte (vgl. Tabelle 3). Trotz durchgängiger - meist zweistelliger Verluste ist die CDU in fast allen Berufs- und Tätigkeitsgruppen weiterhin stärkste Kraft. Ausnahme sind lediglich die Arbeitslosen, bei denen die Linke die meisten Stimmen gewann. Überdurchschnittliche Ergebnisse erzielte die Union bei ihrer traditionellen Klientel, den Selbständigen (allerdings auch hier mit einem großen Minus, das zugunsten der FDP ging) und Rentnern. Auch bei konfessionsgebundenen Wählern schnitt sie sehr gut ab, bei Katholiken vereinte sie trotz eines Minus von 13 Punkten mehr als die Hälfte der Stimmen auf sich. Für die Erzielung eines guten Gesamtergebnisses hilft dies allerdings wenig in einem Bundesland, in dem nur jeder Zehnte der katholischen Kirche angehört ${ }^{19}$, knapp zwei Drittel aber konfessionslos sind.

In dieser großen Gruppe der Konfessionslosen und bei gewerkschaftlich gebundenen Arbeitern erzielte die Linke ihre besten Resultate. Darüber hinaus konnte sie jeden dritten Arbeitslosen von ihrem Politikangebot überzeugen und war bei Rentnern und Arbeitern

19 Vgl. Thüringer Landesamt für Statistik, Thüringer Jahrbuch für Statistik, Erfurt 2009, S. 34. 
ähnlich stark wie die CDU. Die größten Zuwächse verzeichnete die Linke bei Beamten und Thüringern mit mittlerer formaler Bildung.

Die SPD vermochte in allen sozialstrukturellen Gruppen zuzulegen, konnte sich aber nur in wenigen als zweite Kraft positionieren. Die Sozialdemokraten lagen bei Gewerkschaftsmitgliedern, Rentnern und Beamten über ihrem Landesergebnis, aber überzeugten in der Arbeiterschaft sowie bei Arbeitslosen nur rund jeden Sechsten beziehungsweise Achten.

FDP und Grüne weisen auch bei der sozialstrukturellen Zusammensetzung ihrer Wählerschaft bemerkenswerte Parallelen auf: Beide Parteien haben ihre Schwerpunkte bei Wählern mit hohen Schulabschlüssen, bei Angestellten und in Ausbildung befindlichen Personen. Während die Liberalen bei Selbständigen punkteten und sogar besser abschnitten als Linke und SPD, waren die Grünen bei Beamten überdurchschnittlich erfolgreich.

Die NPD konnte überdurchschnittlich stark Arbeitslose und Arbeiter mobilisieren und war dadurch in diesen Gruppen genauso stark oder stärker als FDP und Grüne.

\begin{tabular}{|c|c|c|c|c|c|c|c|c|c|c|c|c|c|c|}
\hline & \multicolumn{2}{|c|}{$\mathrm{CDU}$} & \multicolumn{2}{|c|}{ Die Linke* } & \multicolumn{2}{|c|}{ SPD } & \multicolumn{2}{|c|}{ FDP } & \multicolumn{2}{|c|}{ Grüne } & \multicolumn{2}{|c|}{ NPD } & \multicolumn{2}{|c|}{ FW } \\
\hline & $\%$ & Diff & $\%$ & Diff & $\%$ & Diff & $\%$ & Diff & $\%$ & Diff & $\%$ & Diff & $\%$ & Diff \\
\hline \multicolumn{15}{|l|}{ Bildung** } \\
\hline hoch & 27 & -10 & 28 & -1 & 19 & +3 & 9 & +6 & 11 & +4 & 3 & +2 & 3 & \pm 0 \\
\hline mittel & 30 & -16 & 29 & +5 & 18 & +6 & 8 & +4 & 4 & +1 & 6 & +3 & 5 & +1 \\
\hline niedrig & 40 & -7 & 25 & -1 & 19 & +4 & 4 & +1 & 3 & \pm 0 & 4 & +3 & 4 & +3 \\
\hline \multicolumn{15}{|l|}{ Tätigkeit } \\
\hline Arbeiter & 31 & -12 & 30 & +4 & 16 & +4 & 7 & +4 & 4 & \pm 0 & 7 & +4 & 6 & +1 \\
\hline Angestellte & 29 & -15 & 25 & \pm 0 & 18 & +4 & 10 & +7 & 10 & +4 & 4 & +3 & 4 & \pm 0 \\
\hline Beamte & 30 & -25 & 19 & +7 & 22 & +6 & 8 & +3 & 14 & +9 & 3 & +2 & 4 & +4 \\
\hline Selbständige & 42 & -15 & 15 & +2 & 11 & +3 & 17 & +11 & 7 & \pm 0 & 5 & +4 & 4 & +2 \\
\hline Rentner & 34 & -10 & 31 & +2 & 23 & +4 & 4 & +3 & 3 & +1 & 2 & +1 & 2 & +1 \\
\hline in Ausbildung & 25 & -10 & 21 & -2 & 19 & +5 & 11 & +5 & 15 & +5 & 4 & \pm 0 & 3 & +1 \\
\hline Arbeitslose & 25 & -8 & 34 & -2 & 13 & +3 & 7 & +3 & 4 & \pm 0 & 9 & +6 & 8 & +6 \\
\hline \multicolumn{15}{|l|}{ Gewerkschaft } \\
\hline Mitglieder & 26 & -8 & 33 & +3 & 22 & +2 & 5 & +3 & 6 & \pm 0 & 3 & +2 & 4 & +2 \\
\hline nur Arbeiter & 26 & -9 & 37 & +4 & 19 & +3 & 3 & +1 & 4 & +3 & 4 & +3 & 5 & -1 \\
\hline Nichtmitglieder & 32 & -13 & 27 & +2 & 18 & +4 & 8 & +4 & 6 & +2 & 5 & +3 & 4 & +1 \\
\hline \multicolumn{15}{|l|}{ Konfession } \\
\hline evangelisch & 39 & -11 & 20 & +3 & 19 & +2 & 7 & +4 & 6 & +2 & 3 & +2 & 4 & \pm 0 \\
\hline katholisch & 55 & -13 & 9 & -4 & 13 & +6 & 7 & +4 & 6 & +3 & 3 & +2 & 5 & +4 \\
\hline keine / andere & 22 & -11 & 35 & \pm 0 & 19 & +5 & 8 & +4 & 6 & +2 & 5 & +3 & 4 & +2 \\
\hline \multicolumn{15}{|c|}{$\begin{array}{l}\text { * Bis Landtagswahl 2004: PDS. } \\
\text { ** Hoch = Abitur / Fachhochschule / Universität; mittel = Mittel- / Realschule; niedrig = kein Abschluss } \\
\text { / Volks- / Hauptschule. } \\
\text { Quelle: Wahltagsbefragungen } 2009 \text { und } 2004 \text { von ARD / Infratest dimap. }\end{array}$} \\
\hline
\end{tabular}




\subsection{Wahlverhalten nach Regionen}

Die gebrochene Dominanz der CDU lässt sich nicht nur am Gesamtergebnis, sondern auch auf Wahlkreisebene beobachten. 2004 wurde die CDU in 43 der 44 Wahlkreise stärkste Partei, diesmal waren es nur noch 31. Mit Ausnahme des Wartburgkreises I verlor sie in allen Wahlkreisen. Seit 1990 bestehende Traditionen setzten sich indes weiter fort, denn ihre besten Wahlkreisergebnisse fuhr die Union auch diesmal im katholisch geprägten Eichsfeld im Nordwesten des Landes ein. ${ }^{20}$ Zugleich bedeuteten sie aber auch den größten Absturz in der Wählergunst $(-17,0)$. Deutliche Schwächen hat die CDU generell in den thüringischen Städten, wo sie im Schnitt nur jede vierte Stimme erzielte.

Die Linke wurde in 13 Wahlkreisen stärkste Partei, fünf Jahre zuvor gelang ihr dies nur in zwei Wahlkreisen. In mehr als zwei Dritteln aller Wahlkreise legte sie zu. Wie gehabt schnitt die Linke in Städten beziehungsweise Regionen besonders gut ab, die über eine Industrietradition verfügen, teilweise DDR-Bezirkszentren waren sowie mit Arbeitsmarktund Abwanderungsproblemen kämpfen. Auffallend war im Städtevergleich ihre Schwäche im Raum Jena mit seinen vergleichsweise günstigen Wirtschafts- und Arbeitsmarktdaten.

Die SPD verbuchte in allen 44 Wahlkreisen Stimmengewinne, nachdem sie 2004 überall Verluste zu verzeichnen hatte. Sie war vergleichsweise stärker: einerseits in Nordthüringen und damit in Wahlkreisen mit sichtbaren Strukturproblemen, andererseits aber auch in der thüringischen Leuchtturm-Region Jena, dem Wohnort Matschies. Ihre Schwachpunkte spiegelten weitgehend die Hochburgen von Union und Linken.

Die Liberalen legten ebenfalls in allen Wahlkreisen zu und hatten einen zusammenhängenden regionalen Schwerpunkt im Osten Thüringens. Den größten Zuwachs erzielte die FDP im Unstrut-Hainich-Kreis II $(+5,6)$.

Durchgängige Zuwächse erzielten auch die Grünen, deren Hochburgen in den Universitätsstädten Erfurt, Jena und Weimar zu lokalisieren sind, wo die Partei zweistellige Ergebnisse erzielte. Aber auch außerhalb der Dienstleistungszentren punkteten die thüringischen Grünen: Immerhin blieben sie in der Hälfte aller Wahlkreise über dem Fünfprozentniveau.

Die Region Eichsfeld und der Thüringer Wald gehören zu den regionalen Schwerpunkten der Freien Wähler, in insgesamt zehn Wahlkreisen übersprangen sie die Fünfprozenthürde. Gleiches gilt für die NPD, die ebenfalls in zehn Wahlkreisen 5,0 Prozent der Zweitstimmen oder mehr erreichte.

Der Einbruch der CDU lässt sich auch an den Erststimmenergebnissen ablesen. In den 1990er Jahren gelang es nur zwei Nicht-CDU-Kandidaten, eines der 44 Direktmandate zu erobern. Vor fünf Jahren waren noch 39 direkt gewählte Abgeordnete von der Union. 2009 errang die CDU nur noch 28 Direktmandate, rund ein Drittel ging an die Linke (14) und an die SPD (2).

\subsection{Bewertung der Parteien}

Die Kompetenzzuschreibungen an die Parteien zeigen deutlich, wie die CDU langfristig Vertrauen verloren hat. Zwar wurde zur Wahl 2009 den Christdemokraten in der Arbeits-

$20 \mathrm{Zu}$ den historisch bedingten konfessionellen Besonderheiten im Land siehe Antonio Peter, Thüringen - Im Herzen Deutschlands, in: Hans-Georg Wehling (Hrsg.), Die deutschen Länder, Wiesbaden 2004, S. 297 - 309, S. 299. 
markt-, Wirtschafts- und Bildungspolitik nach wie vor am meisten Kompetenz zugewiesen, sie mussten aber innerhalb der letzten zwei Wahlperioden Vertrauenseinbußen von bis zu 20 Punkten verkraften (Wirtschaft: -20, Arbeit: -13, Bildung: -15 Punkte ${ }^{21}$ ). Bei den wirtschaftsnahen Themen, vor allem in der Steuerpolitik, war der CDU zudem in der FDP eine starke Konkurrenz erwachsen. In der Summe sah nur ein Drittel der Thüringer die wichtigsten Probleme des Landes bei Ministerpräsident Althaus und seiner Partei in guten Händen. Zwar konstatierte jeder zweite Thüringer, dass die Union das Land nach vorn gebracht habe ${ }^{22}$, als soziale Schutzmacht galt sie bei der Bevölkerung jedoch nicht: Sieben von zehn meinten, dass sich mit der CDU die sozialen Ungleichheiten verstärkten und Arbeitnehmerinteressen vernachlässigt würden. Nur für 17 Prozent war die CDU die Partei, die sich für angemessene Löhne einsetzt. Die Kritik fiel folglich massiv aus: Die Arbeit der Regierung wurde - wie bereits 2004 - von einer deutlichen Mehrheit kritisch gesehen. Auch ein Fünftel der eigenen Anhänger war unzufrieden. Die Wechsler von CDU zur SPD und zur Linken waren in erster Linie durch das Thema soziale Gerechtigkeit motiviert. Wirtschaftspolitische Überlegungen waren bestimmend für den Wählerstrom in Richtung FDP.

Die Linke wurde auch vor dieser Wahl vor allem als Partei wahrgenommen, die bestehende Probleme benennt, aber wenig Lösungskonzepte anbieten kann. Mehrheitlich galt sie als Partei des sozialen Ausgleichs und als Vertreterin spezifisch ostdeutscher Interessen. Darüber hinaus war ihr in der Bildungs-, Familien- und Ausländerpolitik sowie beim Einsatz gegen Abwanderung junger Leute eine stärkere Profilierung als 2004 gelungen. In Wirtschaftsfragen und auch bei der Bewältigung der Wirtschaftskrise wurde der Linken vergleichsweise wenig zugetraut. Immerhin rund sechs von zehn Befragten hätten eine Regierungsbeteiligung der Linkspartei begrüßt - allerdings war nur eine kleine Minderheit der Meinung, sie sollte auch den Regierungschef stellen.

Der SPD fiel es wie 2004 schwer, sich inhaltlich zu profilieren. Nur beim Einsatz für angemessene Löhne wurde ihr mehr zugetraut als den anderen Parteien. In der Frage sozialer Gerechtigkeit erhielten die Sozialdemokraten zwar eine größere Kompetenzzuschreibung als 2004, blieben aber dennoch knapp hinter der Linken. Eine Mehrheit sah in der SPD keine politische Alternative zur CDU, zudem wurde ihr im Bund die Reform der Sozialgesetzgebung und die Einführung der Rente mit 67 nachgetragen. In der Folge war aus Sicht der Bevölkerung die SPD keine Partei der „kleinen Leute“ mehr. Von der BundesSPD hatte die Landespartei keine positiven Impulse zu erwarten, da weder der Kanzlerkandidat Frank-Walter Steinmeier selbst noch seine im „Deutschlandplan“ vorgestellten arbeitsmarktpolitischen Ziele die Thüringer überzeugten. Das Dilemma der SPD, sich im Parteienwettbewerb in zwei Richtungen profilieren zu müssen, wird an der Motivstruktur der Wechselwähler deutlich: Wähler, für die soziale Gerechtigkeit das wahlentscheidende Thema war, entschieden sich mehr als doppelt so häufig für die Linke wie für die SPD. Und Wähler, denen ökonomische Themen wichtig waren, votierten auch hier neben der CDU eher für die Linke als für die Sozialdemokraten.

Den Grünen und der FDP kam angesichts offener Machtkonstellationen nach der Landtagswahl eine besondere Bedeutung zu. Die Freidemokraten profitierten zum einen massiv von der Schwäche der thüringischen Union, vermochten sich aber auch selbst in-

21 Vgl. Vorwahlerhebungen 1999, 2004 und 2009 von ARD / Infratest dimap.

22 Zum Vergleich: In Sachsen waren es 70 Prozent, die dieser Aussage zugestimmt haben, siehe Infratest dimap, a.a.O. (Fn. 11), S. 26. 
haltlich stärker zu profilieren als 2004. Am deutlichsten galt das in der Steuer- und der Wirtschaftspolitik. Für knapp jeden Zweiten war die FDP die Partei, die sich auch in der Krise am klarsten für die Marktwirtschaft einsetzt, wobei der Vorwurf der sozialen Kälte die andere Seite der Medaille war. Aus Sicht von 61 Prozent spielte beim Votum für die FDP die Absicht eine Rolle, Rot-Rot in Thüringen zu verhindern. Etwa genauso viele sahen sie wegen ihres marktwirtschaftlichen Kurses als gute Alternative zur Union. Zwei Drittel aller Wahlberechtigten attestierten den Grünen die Rolle als Korrektiv zu den großen Parteien und hätten deren Regierungsbeteiligung begrüßt. Auch mit ihren inhaltlichen Angeboten stieß die Öko-Partei grundsätzlich auf sehr positive Resonanz. Allerdings fiel ihr als außerparlamentarische Opposition eine programmatische Profilierung über ihr Kernthema Umweltpolitik hinaus schwer.

\begin{tabular}{|l|c|c|c|c|c|c|}
\hline Tabelle 4: Parteikompetenzen (in Prozent) \\
\hline & CDU & $\begin{array}{c}\text { Die } \\
\text { Linke }\end{array}$ & SPD & FDP & Grüne & $\begin{array}{c}\text { keine Partei } \\
\text { / weiß nicht }\end{array}$ \\
\hline Wirtschaft voranbringen & $\mathbf{4 2}$ & 6 & 20 & 8 & 2 & 20 \\
Arbeitsplätze sichern und neue schaffen & $\mathbf{3 4}$ & 10 & 22 & 4 & 3 & 25 \\
Gute Schul- und Bildungspolitik & $\mathbf{2 8}$ & 25 & 23 & 3 & 5 & 14 \\
Verschuldung in den Griff bekommen & 25 & 6 & 15 & 7 & 2 & $\mathbf{4 2}$ \\
Gute Steuerpolitik betreiben & 22 & 14 & 21 & 12 & 1 & $\mathbf{2 9}$ \\
Abwanderung junger Leute aus Thüringen & 22 & 15 & 20 & 4 & 3 & $\mathbf{3 2}$ \\
stoppen & 16 & $\mathbf{3 0}$ & 28 & 4 & 4 & 16 \\
Für soziale Gerechtigkeit sorgen & 25 & $\mathbf{2 7}$ & 25 & 2 & 5 & 14 \\
Gute Familienpolitik und Kinderbetreuung & 17 & 21 & $\mathbf{2 8}$ & 4 & 2 & 25 \\
Für angemessene Löhne sorgen & 14 & 5 & 9 & 1 & $\mathbf{5 4}$ & 16 \\
Gute Umweltpolitik betreiben & $\mathbf{3 4}$ & 11 & 21 & 4 & 2 & 26 \\
Wichtigste Probleme des Landes lösen & \\
\hline Anmerkung: Höchster Wert ist fett. & \\
Quelle: Vorwahlerhebung von ARD / Infratest dimap. &
\end{tabular}

\subsection{Bedeutung der Spitzenkandidaten}

Wie bereits 2004 spielte die personelle Aufstellung eine große Rolle für den Ausgang der Wahl. Damals konnte Althaus den Vertrauensverlust der CDU in der Wählerschaft noch durch seine große Popularität auffangen. Dies ist ihm 2009 nicht annähernd gelungen. Die Ursache dafür lag zum einen darin, dass die Spitzenkandidaten von Linke und SPD ihre Bekanntheit deutlich steigerten, zum anderen ging der Ministerpräsident ohne Amtsbonus in die Wahlauseinandersetzung. Mit seiner Arbeit zeigten sich unmittelbar vor dem Wahlgang nur 47 Prozent zufrieden, fast genauso viele bewerteten die Leistung der Herausforderer Matschie und Ramelow positiv (jeweils 44 Prozent). Fünf Jahre zuvor vermochte Althaus noch zwei Drittel aller Bürger von sich überzeugen. Der Amtsinhaber lag auch in der direkten Konfrontation um das Ministerpräsidentenamt nur noch gleichauf mit dem SPD-Chef. Hätten die Thüringer die Wahl zwischen Althaus und Ramelow gehabt, wäre der Vorsprung zugunsten des Amtsinhabers allerdings deutlicher ausgefallen. Im Profilvergleich konnte der CDU-Spitzenkandidat seine Herausforderer in den meisten Bereichen auf Distanz halten. 
Allerdings sahen die Bürger Ramelow wie auch Matschie als Kandidaten, die sich stärker für soziale Gerechtigkeit einsetzen. Bei der Zuschreibung von Glaubwürdigkeit lagen die Herausforderer jeweils gleichauf mit dem Amtsinhaber. Dieses Defizit resultierte offensichtlich aus Althaus' Umgang mit seinem Skiunfall: Die Hälfte der Thüringer war der Meinung, dass er nicht ehrlich und glaubwürdig damit umgegangen sei (48 Prozent) und dass er nach dem Unfall nicht mehr zu vorheriger Leistungsfähigkeit zurück gefunden habe (51 Prozent). Althaus' wenig überzeugender Auftritt beim einzigen Fernsehstreitgespräch mit seinen Herausforderern am Montag vor dem Urnengang konnte diese Sicht nicht ändern. Die Angriffe seiner Herausforderer vermochte der Amtsinhaber nicht zu parieren. ${ }^{23}$ Die Vorbehalte gegenüber Althaus zeigten sich auch bei seinem Wahlkreisergebnis. Zwar erzielte er mit 54,2 Prozent das beste Erststimmenresultat aller Parteien, musste aber gleichzeitig trotz gestiegener Wahlbeteiligung mit 19,9 Punkten auch das größte Minus hinnehmen. Sein Kandidatenbonus - gemessen am Erststimmenplus im Wahlkreis - fiel mit 2,0 Punkten nur durchschnittlich aus.

Die Linke verfügte mit Bodo Ramelow über einen sehr bekannten und recht beliebten Spitzenkandidaten, der auch beträchtliche Teile der SPD- und Grünen-Anhängerschaft von sich überzeugen konnte. Er errang zwar nicht das beste Linke-Erststimmenergebnis ${ }^{24}$, gewann allerdings mit Erfurt III einen bisherigen CDU-Wahlkreis - wenn auch äußerst knapp mit 34 Stimmen.

Der SPD-Spitzenkandidat Christoph Matschie hatte binnen der letzten fünf Jahre deutlich an Statur gewonnen: Er vermochte stärker zu punkten als 2004 und zog bei der Bewertung seiner politischen Arbeit mit Althaus und Ramelow etwa gleich. ${ }^{25}$ Auch Matschie eroberte einen bisherigen CDU-Wahlkreis: Jena I mit 26,9 Prozent. ${ }^{26}$

\section{Regierungsbildung und Oppositionsformierung}

Das Wahlergebnis machte die drittstärkste Partei zur „Königsmacherin“: Eine Regierungsbildung ohne die SPD war rechnerisch und politisch unmöglich. Weil eine schwarz-gelbe

23 In einer von Infratest dimap am 27. August bei 500 thüringischen Wahlberechtigten durchgeführten Umfrage nannten von denen, die das Streitgespräch im MDR-Fernsehen gesehen hatten $(\mathrm{N}=86), 35$ Prozent Ramelow, 28 Prozent Matschie und nur neun Prozent Althaus als denjenigen, der am besten abgeschnitten hat. 25 Prozent antworteten „keiner der drei“. Vgl. auch Philipp Neumann, Ramelow und Matschie Seit' an Seit' gegen Althaus, in: Die Welt vom 25. August 2009, http://www.welt.de/politik/deutschland/article4391045/Ramelow-und-Matschie-Seit-an-Seit-gegen-Althaus.html (Abruf am 25. August 2009); Claus Peter Müller, Thüringer Landesvater in der Defensive, in: FAZ online vom 25. August 2009.

24 Das erreichte Petra Enders im Ilm-Kreis I: Mit 40,0 Prozent Erststimmenanteil übernahm die Großbreitenbacher Bürgermeisterin den bisherigen CDU-Wahlkreis.

25 Dabei hatte Matschie offenbar die örtliche Präsenz als Fraktionsvorsitzender in Erfurt geholfen. Im Vorfeld der letzten Wahl hatte er sich als Staatssekretär im Bundesbildungsministerium mit einer Profilierung auf Landesebene schwer getan, zumal er bei der Erklärung der rot-grünen Sozialreformen in Rechtfertigungsnöte kam. Vgl. dazu Karl Schmitt, a.a.O. (Fn. 1), S. 127.

26 Das beste SPD-Erststimmenergebnis verbucht Matthias Hey im Wahlkreis Gotha II: Mit 40,6 Prozent übernimmt er den bisherigen CDU-Wahlkreis. Mit einem Erststimmenplus von 14,7 Punkten sichert er sich zudem den größten persönlichen Bonus aller Kandidaten bei dieser Landtagswahl. 
Koalition keine Mandatsmehrheit erhalten hatte und ein Bündnis der CDU mit der Linken politisch undenkbar war, blieben drei Alternativen übrig: ein Zusammengehen der CDU mit der SPD oder ein Bündnis aus Linken und SPD eventuell unter Einbezug der Grünen. Die Umfragen vor der Wahl zeigten, dass sich 35 Prozent der Bürger von einer schwarz-roten Koalition positive Impulse für das Land erhofften. Bündnisse der Linken mit der SPD (37 Prozent) beziehungsweise auch mit Beteiligung der Grünen (40 Prozent) betrachteten jeweils rund vier von zehn als gut für das Land. Knapp eine Woche nach der Wahl begannen die Sondierungsgespräche sowohl zwischen Linke und SPD als auch zwischen CDU und SPD.

Die Regierungsbildung wies aus Sicht der SPD große Hindernisse auf, deren Überwindung unmittelbar nach der Wahl unklar schien: Zum einen gab es zwischen der Linken und der SPD zwar in den einzelnen Sachthemen weitgehende inhaltliche Übereinstimmung, die Frage der Regierungsführung war jedoch verfahren. Obwohl nur Chef der drittstärksten Kraft, bekräftigte Matschie noch am Wahlabend seinen Anspruch auf das Amt des Regierungschefs. ${ }^{27}$ Zum anderen war die SPD im Wahlkampf für eine Ablösung des „Systems Althaus" eingetreten, so dass ernsthafte Gespräche zur Regierungsbildung mit dem amtierenden Ministerpräsidenten unmöglich erschienen.

Der politische Druck auf Althaus war in den Tagen nach der Wahl gewachsen, weil die SPD bei der Forderung nach seiner Ablösung blieb. Er galt somit als Haupthindernis für die Bildung einer schwarz-roten Landesregierung. Nachdem Althaus selbst am Wahlabend und in den Tagen danach seinen Amtsverzicht noch ausgeschlossen hatte, trat er am 4. September überraschend als Ministerpräsident und CDU-Landesvorsitzender zurück. In diese Entscheidung waren offenbar weder seine engsten politischen Vertrauten in Erfurt noch die Parteiführung in Berlin eingebunden gewesen. ${ }^{28}$ Althaus eröffnete durch seinen Rücktritt erst eine realistische Verhandlungsoption für ein Zusammengehen mit der SPD, so dass selbst Angela Merkel über sein Ausscheiden erleichtert schien. ${ }^{29}$ Seinen Platz in der Sondierungskommission nahm Sozialministerin Christine Lieberknecht ein. Als die Staatskanzlei am Montag darauf mitteilte, dass Althaus die Kabinettssitzungen weiter leiten werde - wie es die Landesverfassung vorsieht - schlug die stellvertretende Regierungschefin Birgit Diezel am Abend Lieberknecht als Ministerpräsidentin einer möglichen CDU/SPD-Koalition vor. ${ }^{30}$ Lieberknecht erklärte am Morgen darauf in einem Radio-Interview die Ära Althaus für beendet.

27 Dass der nach Sitzen kleinere Partner in einer Koalition den Ministerpräsidenten stellt, ist in der Bundesrepublik zwar ungewöhnlich, gleichwohl nicht ohne historisches Beispiel: Der erste baden-württembergische Ministerpräsident war Reinhold Maier von der FDP. Maier bildete eine Landesregierung aus seiner mit 23 Mandaten ausgestatteten liberalen Fraktion, der mit 38 Sitzen deutlich größeren SPD-Fraktion und dem BHE. Vgl. dazu Ulrich Eith, Das Parteiensystem Baden-Württembergs, in: Uwe Jun / Melanie Haas / Oskar Niedermayer (Hrsg.), Parteien und Parteiensysteme in den deutschen Ländern, Wiesbaden 2008, S. 103 - 124, S. 109.

28 Althaus erklärte später, dass seine Rücktrittsentscheidung bereits am Wahlabend festgestanden habe. Vgl. dazu Interview mit Dieter Althaus, „Es tut noch weh“, in: Thüringer Allgemeine vom 16. Dezember 2009, S. 4 f.

29 Vgl. Georg Paul Hefty, Kleine Große, in: FAZ vom 4. September 2009, S. 1.

30 Es kursierten zudem Gerüchte, dass Althaus Fraktionschef Mohring als neuen Landesvorsitzenden vorschlagen wollte. Siehe Claus Peter Müller, Althaus verteidigt sein Vorgehen, in: FAZ vom 9. September 2009, S. 4. 
Nach der ersten Sondierungsrunde von Linken und SPD verständigten sich die beiden Parteien darauf, die Grünen zu den Verhandlungen hinzuzuziehen, weil ihnen angesichts schwieriger Entscheidungen die eigene Mehrheit von nur einem Sitz nicht ausreichte. ${ }^{31}$ Die Grünen zeigten sich offen für Gespräche, reagierten aber zugleich skeptisch. ${ }^{32}$ Nach der ersten Dreier-Runde beschlossen sie, auf einem Sonderparteitag über den Eintritt in Sondierungsgespräche zu entscheiden.

Angesichts der schwierigen Frage, wer in einem rot-rot-grünen Bündnis das Ministerpräsidentenamt übernehmen sollte, kursierten Gerüchte, die prominente Politiker der SPD und der Grünen als Kandidaten ins Spiel brachten. ${ }^{33}$ Einen Tag vor der Entscheidung des Grünen-Parteirates über den Eintritt in die Sondierungsgespräche plädierte Ramelow für einen gleichberechtigten Personalvorschlag aller drei Parteien, was als sein Verzicht auf das Amt interpretiert wurde. ${ }^{34}$ Insbesondere von der SPD wurde dies mit Erleichterung aufgenommen. Der Parteirat der Grünen entschied mit großer Mehrheit für Sondierungsgespräche, aber gegen einen Linken-Politiker als Ministerpräsidenten. ${ }^{35}$ Gleichwohl zielte Ramelows Vorstoß nur vordergründig in Richtung Grüne, in erster Linie wollte er damit wohl eher die Präferenzen der SPD-Basis auf eine Koalition mit der Linkspartei lenken. Die erste Dreier-Runde wurde danach von grüner Seite skeptisch bewertet, auch weil aus deren Sicht die Stimmung zwischen SPD und Linke angespannt sei. ${ }^{36}$ Den parallel laufenden Austausch zwischen Schwarz-Rot beschrieben beide Seiten hingegen als konstruktiv.

Die Bundestagswahl Ende September gab den Gesprächen eine besondere Dynamik, weil die SPD dabei als einzige der fünf Thüringer Parlamentsparteien Stimmenanteile verloren hatte - und das sogar zweistellig. ${ }^{37}$ Bereits während der Sondierungsphase hatte sich innerhalb der SPD Protest gegen ein Zusammengehen mit der CDU formiert. ${ }^{38}$ Der Erfurter SPD-Oberbürgermeister Andreas Bausewein formulierte wenige Tage nach der Wahl: „Ich kann mir nicht ernsthaft vorstellen, dass die SPD in Thüringen nach diesem Bundestagswahlergebnis in eine Große Koalition hineingehen wird. "39 Während Matschies Kritiker den Landesvorsitzenden als größtes Hindernis für eine linke Koalition ansahen, versuchte dieser deutlich zu machen, dass es ihm nicht um seine Person gehe und erklärte seinen persönlichen Verzicht auf das Ministerpräsidentenamt - allerdings gab er den Anspruch nicht auf,

31 Interview mit Bodo Ramelow, Eine Stimme mehr reicht mir nicht, in: Tagesspiegel vom 14. September 2009, S. 4.

32 Siehe Äußerungen der Grünen-Vorsitzenden Astrid Rothe-Beinlich, zitiert nach: SZ, Schwierige Partnersuche, 05./06. September 2009, S. 6.

33 Genannt wurden unter anderem die Bundestagsvizepräsidentin Katrin Göring-Eckardt und die Politikprofessorin Gesine Schwan.

34 Vgl. Philipp Neumann, Ramelows Rückzug verärgert die Linke, in: Die Welt vom 19. September 2009, S. 4.

35 Vgl. Christiane Kohl, Umworbene SPD, in: SZ vom 21. September 2009, S. 6.

36 Vgl. Thüringer Grüne skeptisch, in: FAZ vom 22. September 2009, S. 4; CDU will Diezel als Präsidentin des Erfurter Landtags, in: FAZ vom 24. September 2009, S. 4.

37 Ergebnis der Bundestagswahl vom 27. September 2009 in Thüringen: CDU, 31,2 Prozent $(+5,5)$, Linke 28,8 (+2,7), SPD 17,6 (-12,2), FDP 9,8 $(+1,9)$, Grüne 6,0 $(+1,2)$.

38 Die Proteste wurden angeführt von den SPD-Oberbürgermeistern von Erfurt und Gera, Andreas Bausewein und Norbert Vornehm.

39 Interview mit Andreas Bausewein, $\mathrm{Zu}$ weit in die Mitte gerutscht, in: Thüringer Allgemeine vom 30. September 2009, S. ER4 (Lokalausgabe Erfurter Allgemeine). 
dass ein SPD-Politiker dieses Amt bekleiden sollte. Am 30. September wurde zum letzten Mal zwischen Linken, SPD und Grünen auf der einen sowie CDU und SPD auf der anderen Seite sondiert. Die Gespräche führten aus Sicht der SPD nicht dazu, dass die Linke einen sozialdemokratischen Ministerpräsidenten akzeptieren würde. Am Ende war, trotz großer programmatischer Übereinstimmung, der fehlende Kooperationswillen zwischen den bestimmenden Personen Ramelow und Matschie ${ }^{40}$ wohl ausschlaggebend für das Scheitern eines linken Bündnisses. Am Abend desselben Tages beschloss der SPD-Landesvorstand daher mehrheitlich die Aufnahme von Koalitionsverhandlungen mit der CDU. ${ }^{41}$

Die Entscheidung löste teils heftige Kritik aus. Die Reaktionen fanden ihren Höhepunkt in dem Vorwurf einer Missachtung des Wählerwillens und „Wählerbetrugs“ (LinkeLandesvorsitzender Knut Korschewsky) sowie in der Verunglimpfung Matschies als „politischen Scharlatan“ (Ex-SPD-Landesvorsitzender Richard Dewes) ${ }^{42}$. Die SPD-interne Kritik verebbte allerdings in Folge einer Basisversammlung am 10. Oktober und des direkten Dialogs Matschies mit seinen innerparteilichen Gegnern sowie mit dem Beschluss, den Landesparteitag 2010 mit Vorstandswahlen um drei Monate auf März vorzuziehen. ${ }^{43}$ So stimmten auf dem SPD-Parteitag am 25. Oktober 148 von 199 Delegierten für den Koalitionsvertrag mit der CDU. Bei dem nahezu zeitgleich abgehaltenen Parteitag der CDU votierten deren 133 Delegierte geschlossen für das Vertragswerk. Christine Lieberknecht wurde bei dieser Versammlung mit 83,3 Prozent zur neuen Landesvorsitzenden gewählt.

Ende Oktober unterzeichneten Lieberknecht und Matschie die Koalitionsvereinbarung, bei der die CDU der SPD weit entgegengekommen war. Dies betrifft die Einführung einer Gemeinschaftsschule, bei der die Kinder bis zur 8. Klasse gemeinsam lernen (allerdings lediglich als freiwilliges Angebot), den Anspruch auf einen Kindergartenplatz ab dem ersten (bisher zweiten) Geburtstag, den Ausschluss von Studiengebühren und die Wiedereinführung der Stichwahl auf kommunaler Ebene. Allerdings konnten sich die Sozialdemokraten mit der von ihnen geforderten Abschaffung des umstrittenen Landeselterngeldes und einer Gebietsreform gegen die CDU nicht durchsetzen. Der SPD gelang es aber, die Hälfte der acht Ministerien für sich zu beanspruchen.

Die Wahl der Ministerpräsidentin am 30. Oktober brachte eine handfeste Überraschung, denn Lieberknecht verpasste in den ersten beiden Wahlgängen die Mehrheit der Abgeordnetenstimmen und wurde erst im dritten Wahlgang zur Ministerpräsidentin gewählt - die zweite in Deutschland. Sie erreichte zunächst nur 44 der 48 Stimmen von CDU und SPD und damit eine weniger als die von der Landesverfassung geforderte Mehrheit. Gleiches wiederholte sich beim zweiten Wahlgang - Erinnerungen an das Scheitern

40 Beschrieben als „wochenlange Kabale“, vgl. Elmar Otto, Kabale ohne Liebe beim Showdown der Alphatiere, in: Thüringer Landeszeitung vom 30. September 2009, S. 4.

41 Die Entscheidung fiel mit 18 zu sechs Stimmen, vgl. Volkhard Paczulla, Regierung soll in vier Wochen stehen, in: Ostthüringer Zeitung vom 2. Oktober 2009, S. 1.

42 Vgl. Christiane Kohl, Thüringer SPD rebelliert gegen Schwarz-Rot, in: SZ vom 2./3./4. Oktober 2009, S. 1.

43 Am 6. März 2010 wurde Matschie ohne Gegenkandidaten auf dem Landesparteitag in Ilmenau mit einer Mehrheit von 78,4 Prozent der abgegeben Stimmen als Landeschef wiedergewählt. Damit übertraf er das Ergebnis der Wahl von 2008 leicht. Bausewein kehrt als stellvertretender Vorsitzender der Landespartei in den Landesvorstand zurück. 
von Heide Simonis 2005 wurden wach. ${ }^{44}$ Vor dem dritten Wahlgang, bei dem nur eine einfache Mehrheit notwendig ist, erklärte Bodo Ramelow seine Kandidatur. Dies disziplinierte offenbar die Koalitionsabgeordneten, denn in dieser Abstimmung erhielt sie 55 Voten - die sieben FDP-Abgeordneten stimmten ebenfalls für Lieberknecht. Ramelow selbst bekam 27 Stimmen, nur eine mehr als Linke-Abgeordnete im Haus waren (einer fehlte an diesem Tage). Dass sich nur ein Abgeordneter zusätzlich für ihn entschied, vermutlich von den Grünen, ist als Ausdruck der Skepsis gegenüber dem Spitzenmann der Linken zu sehen. Fünf Abgeordnete enthielten sich. Lieberknecht hatte offenbar bereits im Vorfeld Heckenschützen gefürchtet und deshalb die Benennung der Ministerriege auf die Folgewoche verschoben, um Denkzettel enttäuschter Fraktionsmitglieder zu vermeiden. Zwei Monate nach der Wahl wurden am 4. November die Minister vereidigt - noch nie hatte eine Regierungsbildung in Thüringen länger gedauert.

\begin{tabular}{|l|l|}
\hline Tabelle 5: Das thüringische Kabinett nach der Landtagswabl 2009 \\
\hline Ministerpräsidentin & Christine Lieberknecht (CDU)* \\
\hline $\begin{array}{l}\text { Minister für Bildung, Wissenschaft und Kultur und stellver- } \\
\text { tretender Ministerpräsident }\end{array}$ & Christoph Matschie (SPD)** \\
\hline Innenminister & Prof. Dr. Peter Michael Huber (CDU)** \\
\hline Justizminister & Dr. Holger Poppenhäger (SPD)** \\
\hline Finanzministerin & Marion Walsmann (CDU)* \\
\hline Minister für Wirtschaft, Arbeit und Technologie & Matthias Machnig (SPD)** \\
\hline Ministerin für Soziales, Familie und Gesundheit & Heike Taubert (SPD)** \\
\hline $\begin{array}{l}\text { Minister für Landwirtschaft, Forsten, Umwelt und } \\
\text { Naturschutz }\end{array}$ & Jürgen Reinholz (CDU)* \\
\hline Minister für Bau, Landesentwicklung und Verkehr & Christian Carius (CDU)** \\
\hline $\begin{array}{l}\text { Minister für Bundes- und Europaangelegenheiten und Chef } \\
\text { der Staatskanzlei }\end{array}$ & Dr. Jürgen Schöning (parteilos) ${ }^{* *}$ \\
\hline $\begin{array}{l}* \text { Neu in dieser Funktion. } \\
* *\end{array}$ & \\
\hline
\end{tabular}

Bei allen Parteien standen wichtige Personalwechsel an: Die frühere CDU-Finanzministerin Birgit Diezel wurde zur Landtagspräsidentin gewählt. Während Lieberknecht neue Parteivorsitzende ist, bleibt Mike Mohring CDU-Fraktionschef. Dieter Althaus, zunächst einfacher Landtagsabgeordneter, schied am 30. April 2010 aus dem Landtag aus und wechselte als Lobbyist zu einem österreichisch-kanadischen Automobilzulieferer. Bodo Ramelow ist neuer Fraktionschef der Linken. ${ }^{45}$ Nachfolger Matschies als Vorsitzender der SPD-Fraktion wurde deren Parlamentarischer Geschäftsführer Uwe Höhn. Der liberale Spitzenkandidat Uwe Barth ist Vorsitzender der FDP-Fraktion. Die Grünen-Fraktion bestimmte Anja Siegesmund zu ihrer Vorsitzenden, Spitzenkandidatin Rothe-Beinlich wurde zu einer der Vize-Präsidenten des Landtags gewählt. Neue Sprecher des grünen Landesverbandes sind Madeleine Henfling und Dieter Lauinger.

44 Vgl. dazu Thomas Saretzki / RalfTils, Die schleswig-holsteinische Landtagswahl vom 20. Februar 2005: Geheime Stimmverweigerung für Ministerpräsidentin Heide Simonis erzwingt Große Koalition, in: ZParl, 37. Jg. (2006), H. 1, S. 145 - 163.

45 Diese Funktion hatte Ramelow bereits von 2001 bis 2005 inne. 


\section{Zusammenfassung}

(1) Der 30. August 2009 war für die Thüringer CDU ein Wahltag mit historischer Dimension: Nach zehn Jahren Alleinregierung kam sie nur auf 31,2 Prozent der Zweitstimmen, ihr schlechtestes Ergebnis. Die zwei Jahrzehnte dauernde Dominanz der CDU war damit beendet. Die Linke verbesserte sich auf ein neues Rekordergebnis (27,4 Prozent). Die SPD legte auf 18,5 Prozent zu und blieb drittstärkste Kraft. FDP (7,6 Prozent) und Grüne (6,2 Prozent) gelang nach 15 Jahren wieder der Einzug in den Landtag. Die Wahlbeteiligung war mit 56,2 Prozent erstmals seit 1994 wieder angestiegen.

(2) Der langfristige Vertrauens- und Ansehensverlust der CDU konnte bei der letzten Wahl noch durch die große Popularität von Dieter Althaus aufgefangen werden - 2009 gelang dies nicht mehr, denn der Ministerpräsident ging ohne Amtsbonus in den Wahlkampf. Der Umgang mit seinem Skiunfall am Neujahrstag, bei dem eine Frau starb, irritierte die Wahlbevölkerung und die eigene Partei. Althaus' fehlendes Bekenntnis zu seiner Schuld an dem Unglück und dessen Instrumentalisierung durch ihn selbst schadeten seinem Ansehen massiv. Außerdem hatte die CDU in den vergangenen zehn Jahren in den wichtigen Bereichen Arbeitsmarkt-, Wirtschafts- und Bildungspolitik stetig an Kompetenzzuschreibung verloren.

(3) Die Linke hatte sich neben ihrer Rolle als Partei des sozialen Ausgleichs und Vertreterin spezifisch ostdeutscher Interessen in anderen Politikfeldern stärker profiliert. Auch auf personeller Ebene war sie mit Bodo Ramelow gut aufgestellt. Ihre Hoffnung, in Thüringen ihren ersten Ministerpräsidenten stellen zu können, erfüllte sich jedoch nicht. Trotz größerer inhaltlicher Übereinstimmung mit der Linken entschied sich die SPD für ein Bündnis mit der CDU, denn die Wahl eines Ministerpräsidenten aus der Linken hatte SPD-Chef Christoph Matschie vor der Wahl ausgeschlossen.

(4) Die SPD konnte sich trotz des mäßigen Ergebnisses als Wahlgewinner fühlen, denn ihr fiel die Rolle des „Königsmachers“ zu. Inhaltlich hatten die Sozialdemokraten Schwierigkeiten, sich sowohl gegenüber der CDU als auch der Linken zu profilieren. Ihr Spitzenkandidat Matschie hatte allerdings an Statur gewonnen. Er führte die SPD nach elf Jahren wieder in Regierungsverantwortung, als Juniorpartner einer personell runderneuerten CDU. An ihrer schwierigen Position im Parteiensystem, eingekeilt zwischen CDU und Linke, hat sich indes nichts Grundlegendes geändert.

(5) Die FDP verdoppelte ihre Wählerstimmen auf 7,6 Prozent. Sie profitierte massiv von der Schwäche der Union, vermochte sich aber auch inhaltlich - vor allem in der Steuer- und Wirtschaftspolitik - stärker zu profilieren. Dabei half den Liberalen ihr Image als Partei, die sich auch in der Krise am klarsten für die Marktwirtschaft einsetzt.

(6) Die Grünen erreichten 6,2 Prozent. Mit ihren inhaltlichen Angeboten stießen sie grundsätzlich auf positive Resonanz, eine deutliche programmatische Profilierung über ihr Kernthema Umweltpolitik hinaus fiel ihnen aber schwer.

(7) Die Wahlbeteiligung stieg auf 56,2 Prozent $(+2,4)$. Grund dafür war der mehrheitlich verbreitete Wunsch nach einem politischen Wechsel, gepaart mit der Aussicht, eine Neuordnung der politischen Verhältnisse im Land auch tatsächlich bewirken zu können.

(8) Nach dem Rücktritt von Althaus führte die drittplatzierte SPD sowohl mit der CDU als auch mit der Linken und den Grünen Sondierungsgespräche. Nach heftiger innerparteilicher Kritik setzte Matschie ein Zusammengehen mit der CDU auf einem Parteitag 
durch. In dem Vertragswerk kam die CDU den Sozialdemokraten in vielen Punkten entgegen. Zudem erhielten sie vier der acht Ministerien. Ob die SPD am Ende der Wahlperiode als Juniorpartner der Verlierer des schwarz-roten Bündnisses sein wird, weil die Erfolge der Regierung wie 1999 der CDU zugeschrieben werden, ist offen. In den Verhandlungen mit der CDU ist es der SPD jedenfalls gelungen, eine ganze Reihe ihrer Positionen im Koalitionsvertrag festzuschreiben. Falls sie diese tatsächlich in der Regierungsarbeit der nächsten fünf Jahre umzusetzen und zu kommunizieren vermag, stehen die Chancen der Sozialdemokraten zur Profilierung innerhalb der Koalition recht gut.

(9) Überraschend benötigte Christine Lieberknecht drei Wahlgänge im Parlament, um die zweite Ministerpräsidentin Deutschlands zu werden. In den ersten beiden Wahlgängen fehlten ihr jeweils vier Stimmen aus den Regierungsfraktionen. Erst als im dritten Wahlgang Ramelow für die Linke antrat, bekam Lieberknecht alle Stimmen von CDU und SPD sowie von der FDP. Sie will auf Transparenz und Offenheit in der politischen Arbeit setzen und damit einen neuen Politikstil in Thüringen prägen.

\title{
Die sächsische Landtagswahl vom 30. August 2009: Sachsens Vorreiterrolle für den Bund
}

\author{
Eckhard Jesse
}

\section{Die Ausgangslage}

Die Ausgangslage war 2009 ganz anders als bei den Wahlen zuvor. Zum ersten Mal konnte die Sächsische Union nicht als alleinige Regierungspartei in den Wahlkampf ziehen. Unter der Ägide von Kurt Biedenkopf hatte die CDU mit 53,8 Prozent, 58,1 Prozent und 56,9 Prozent 1990, 1994 und 1999 sensationelle Ergebnisse errungen ${ }^{1}, 2004$ auf dem Höhepunkt der öffentlichen Kritik an Hartz IV unter dem wenig charismatischen Ministerpräsident Georg Milbradt hingegen 15,8 Punkte verloren. Waren bis dahin drei Fraktionen im Parlament vertreten, wurden es nun sechs. Die rechtsextremistische NPD erreichte mit 9,2 Prozent fast so viele Stimmen wie die SPD (9,8 Prozent) und deutlich mehr als die Liberalen (5,9 Prozent) und die Grünen (5,1 Prozent). ${ }^{2}$ Da ein kleines schwarz-gelbes Bündnis knapp an der Arithmetik scheiterte, kam - paradox genug - nur eine kaum größere schwarz-rote Koalition in Frage.

1 Vgl. Ulrich Brümmer, Parteiensystem und Wahlen in Sachsen, Wiesbaden 2006; Christian Demuth / Jakob Lempp (Hrsg.), Parteien in Sachsen, Dresden / Berlin 2006; Anja Mays, Bundespolitische Effekte oder regionale Besonderheiten? Zum Einfluss der Bundespolitik auf die sächsischen Landtagswahlen, in: Kerstin Völkl / Kai-Uwe Schnapp / Everhard Holtmann / Oscar W. Gabriel (Hrsg.), Wähler und Landtagswahlen in der Bundesrepublik Deutschland, Baden-Baden 2008, S. 361 - 380; Gero Neugebauer, Das Parteiensystem Sachsens, in: Uwe Jun / Melanie Haas I Oskar Niedermayer (Hrsg.), Parteien und Parteiensysteme in den deutschen Ländern, Wiesbaden 2008, S. $387-408$.

2 Vgl. Eckhard Jesse, Die sächsische Landtagswahl vom 19. September 2004: Debakel für CDU und SPD gleichermaßen, in: ZParl, 36. Jg. (2005), H. 1, S. $80-100$. 